\title{
Preparing for inclusive consultation, research and policy development: insights from the field of Deafblindness
}

\author{
Alana Roy \\ University of Melbourne \\ Keith R. McVilly \\ University of Melbourne \\ Beth R. Crisp \\ Deakin University
}

\begin{abstract}
There is a paucity of research and guidance on good practice for engaging with people who are Deafblind in policy development and the co-design of services and service systems. Although there are a range of inclusive research methodologies, such as the World Café, Deafblind people are yet to be engaged authentically and effectively in these conversations. To address this issue, and as part of a program of research to investigate and test good practice in this field, we analysed data from semi-structured interviews with eight Deafblindness professionals using inductive thematic analysis. Six themes emerged from the interview data. These were: (1) acknowledging the unique Deafblind world view; (2) the interplay of vulnerability and trusting relationships; (3) the importance of specialist knowledge, skills, and cultural sensitivities; (4) power to the people - addressing power imbalances inherent in consultation processes; (5) same but different - the Deafblind experience of being a culturally and linguistically diverse (CALD) community, and; (6) a challenge that must be met - the imperative to involve people who are Deafblind in research and policy development. If people who are Deafblind are to be engaged in research and policy development, there are a number of guiding principles that need to be considered and practical actions to be taken. These principles include employing a culturally sensitive and trusted research team to spend time working with Deafblind people to help prepare and educate them for research and consultation processes. In so doing, people with lived experiences and professionals in the field can build the trust, knowledge and skills needed to enable authentic co-design and co-production of policy and practice.
\end{abstract}

Keywords: Deafblind, World Café, Research Methods, inclusive research

People from culturally and linguistically diverse (CALD) communities have found themselves on the margins of the social policy agenda and are arguably under-represented in research informing such agenda (Buckmaster \& Thomas, 2009; Hammel et al., 2008; Saunders, 2013). This isolation has contributed to poor outcomes with respect to their health and education, together with their social, economic and political participation in the community more broadly (Australian Institute of Health and Welfare, 2014; Bonevski et al., 2014). One such CALD community comprises those who are Deafblind (Barrett, 1992; Bloeming-Wolbring et al., 2012; Bodsworth, Clare \& Simblett, 2011).

Corresponding author: Alana Roy (alana.roy@unimelb.edu.au) 
Being Deafblind has been recognized by the European Union as a "separate and distinct disability" (European Parliament, 2004) that describes an experience and identity that is not simply the combination of vision and hearing impairments. There are more than 70 different aetiological causes of Deafblindness, with some giving rise to a life-long experience and others manifesting as a deterioration of the senses across the lifespan (Wahlqvist, Möller, Möller \& Danermark, 2013; Wittich, Watanabe \& Gagné, 2012).

The term 'Deafblind' (one word with a capital D) is representative of those individuals, organisations and services who celebrate and promote Deafblind culture and sign language (Ascheman, 2012; MacDonald, 1985). Although, it should be noted that many people who have a combined vision and hearing loss may not use sign language or identify as being 'Deafblind'. However, in saying this, combined vision and hearing loss typically gives rise to the need for highly specialised support services which are commonly delivered from within the Deafblind community.

The Deafblind community represents a unique, yet diverse cultural group within society. Deafblind people have their own language (usually a combination of sign language, tactile sign, spoken language, and braille), as well as their own social norms with respect to interpersonal proximity and touch (Brennan, Su \& Horowitz, 2006; Heine \& Browning, 2002). They present with their own unique orientation and mobility needs using a combination of guide dogs, long canes, digital way finding technology and personal assistants. Some use hearing aids or cochlear implants, others do not. However, their common experience of a combination of impairments to both hearing and vision influence their experience of, and world view concerning, the society in which they live (Barrett, 1992; Berry, Kelley-Bock \& Reid, 2008; Blumsack, 2009).

As is the case for other CALD groups, there is a growing imperative in research and policy development to shift the focus from conducting 'research on' or developing 'policy about' people who are Deafblind, to a focus on research and policy development that is inclusive of people who are Deafblind (Selepak, 2008). Informing this inclusion agenda has been a number of social movements including feminism, the civil rights movement, and more recently the Lesbian, Gay, Bisexual, Transgender, Queer and Intersex (LGBTQI) rights movement (Hillier, Turner \& Mitchell, 2005; Shakespeare, 2006). Consequently, there is growing recognition of the need to develop the community's understanding of those who have traditionally been on the margins, and in turn take the action needed to promote and realise an authentically inclusive society (Suanders, 2013).

Although there is growing agreement among researchers and policy makers that people from CALD backgrounds and those with the lived experience of disability, such as those who are Deafblind, should be included in the formation of public policy, there is a dearth of literature (both peer reviewed and grey literature) to guide such practice (Bruce \& Parker, 2012; Mathos, Lokar \& Post, 2011). Research and consultation processes commonly rely on methodologies that assume participants have full use of all their senses and that they are part of a hearing and sighted world (Heine \& Browning, 2014; Vernon, 1982, 2010). In modern times, C. West Churchman coined the term 'wicked problem' to describe complex and multifaceted problems that are perceived as difficult or impossible to solve, such as those found in economic, environmental, political, climate change and social justice issues (Churchman, 1967; Ferlie, McGivern, Fitzgerald, Dopson \& Bennett, 2011). Rittel \& Webber (1984) argue that when 'wicked problems' are clearly identified meaningful policy can be designed to address social issues.

In the field of disability and culturally and linguistically diverse practice, effective engagement with those who are Deafblind emerges as a 'wicked problem' for researchers and policy makers due to the complex and multifaceted social, political, and economic barriers as they attempted to navigate the world with limited, or no sight and hearing. Deafblindness can limit 
choices and opportunities in accommodation, education and vocational activities. It can confound personal care, orientation, mobility and safety, and negatively impact communication and social relationships. As a consequence of these multiple impairments and limitations, Deafblindness can have a major detrimental effect on a person's social inclusion and access to appropriate services and supports, their quality of life and wellbeing and, consequently their mental health (Heine \& Browning, 2002, 2014; Vernon, 2010).

In an effort to address the imperative to be inclusive in research and policy development processes within the disability research and policy community, there is a growing emphasis on co-design and co-production. Co-design approaches grew out of the manufacturing, building (architectural) and computer software sectors, where there was a growing recognition of the value of involving a variety of stakeholders, including end users, in the production process. Co-production was coined in the 1970's by an American economist, Ostrom, as a result of strained relationships between communities and police in the United States of America (USA). Co-production emerged as an approach to engage communities in consultation, policy and service delivery (Ostrom, 1978). These principles have since been adopted in the social policy and human service sectors. They are claimed to democratise the design and delivery of services, and to harness the expertise of the lived experience of end users (Needham \& Carr, 2009; Pawlikowska, Leach, Lavallee, Charlton \& Piercy, 2007). Codesign is understood to be characterised by being inclusive of all stakeholders and recognising a range of expertise. The processes are inherently respectful and involve negotiation. They seek to maximise participation through conversations that are open, empathic and responsive to all stakeholders (Alford, 2014; Needham \& Car, 2009; Sharp, 1980). Ideas are constantly evaluated and refined through the interaction of all participants. Processes of co-design are outcome focused and aim to generate practical solutions that have ecological validity (Jasanoff, 2004; Muñoz-Erickson, 2014; Wyborn, 2015). Similarly, co-production involves the engagement of people with lived experience in the design and delivery of policy and services (Bovaird \& Loeffler, 2010). However, how co-design and co-production might be progressed with those who are Deafblind remains undeveloped.

The importance of engaging people with lived experience in 'community conversations' as a methodology is gaining popularity in the literature and has been used across a range of CALD groups and people with disabilities (Bumble, Carter, McMilllan, Manikas \& Bethune, 2018; Carter, Sweedenn, Walker \& Moss, 2012; Dutta, 2007). Community conversations are asserted to 'give power to the people' and harnesses the dynamic interaction of people with expertise based on their lived experience, and to generate solutions across a diverse range of real world problems (Carter, Austin \& Trainor, 2012; Minkler \& Wallerstein, 2012; Trainor, 2018). Focus groups are a popular methodology that are known to be inclusive. Focus group methodology grew out of the field of consumer psychology and market research; where there was a need to understand the perspectives and priorities of a population, and what motivated people to act in certain ways. It is a qualitative approach to research in which people, selected as being representative of a larger population, discuss their opinions in an interactive way with others, giving rise to situations in which their opinions can be supported or challenged by their peers, and refashioned in the light of new information provided by other members of the group. Focus groups can be moderated or un-moderated (self-moderated), and can be conducted in face-to-face or on-line formats (Bilson, 2004; Pranee, 2016).

Focus groups are commonly used in research settings to gather new knowledge, seek perspectives, and evaluate services and programs (Beyea \& Nicoll, 2000; Gulanick \& Kenough, 1997; Simm 1998). Furthermore, focus groups, when appropriately adapted to accommodate the needs of participants, have proven to be an efficacious methodology for eliciting the views of people who are culturally and linguistically diverse and those who may have varying communication, literacy, and social needs (Clark et al., 2003). 
In addition, there is a growing emphasis in the social sciences on the application of Appreciative Inquiry (Al). This epistemological perspective, as a counterpoint to more traditional problem focused approaches to inquiry, asks 'what currently works', 'what form might it take if it was to be better than it currently is', and 'how might we achieve this enhanced / improved experience' (Hung et al., 2018; Merier \& Geldenhuys, 2017). These questions are well suited to community conversations involving people with expertise arising from their lived experience. As an extension of the more common focus group approach and the application of appreciative inquiry in the facilitation of community conversations is most evident in World Café (WC) methodology (Brown \& Isaacs, 2005; Carlson, 2011). WC is a process originally developed in the mid 1990s by Juanita Brown and David Isaacs. WC methodology is underpinned by principles of $\mathrm{Al}$; that is, WC seeks to generate collective insights by amplifying the positive, socially shared and constructed realities of participants with the view to generating solutions to wicked problems on a consensus basis (Brown \& Issacs, 2005).

WC was designed for facilitating multi-group discussion and consensus decision-making of the kind envisaged for the series of workshops proposed for the current study. Therefore, it was considered that the WC methodology could be an effective way of bring ing Deafblind people together to share their lived experiences, as at it shares similar benefits to those of focus groups, but with the added benefit of increasing the dynamic interaction among participants. WC can be utilised as a "powerful social technology for engaging people in conversations that matter offering an effective antidote to the fast-paced fragmentation and lack of connection in today's world" (Brown, 2018). People from a range of CALD groups and people with disabilities have been successfully engaged in community conversations, including in WCs, across complex and multifaceted issues (MacFarlane et al., 2017; Schieffer, Issacs \& Gyllenpalm, 2004). WCs have been utilised to share collective intelligence and generate solutions across a range of issues in direct practice, policy, organizations context and social inclusion (Brown, Isaacs \& Tan, 2008; Burke \& Sheldon, 2010; Fallon \& Bean, 2014; Horvath, 2005). However, to date the Deafblind community in Australia and internationally have not yet engaged in such community conversations. Such an approach might, if adapted to the unique needs of those who are Deafblind, provide a useful approach to fostering their inclusion in research and policy development.

Subsequently, as part of our programme of research, we proposed to investigate how the principles of co-design and co-production, and the practices associated with appreciative inquiry, such as World Café, might support and enable the inclusion of people who are Deafblind in research and policy development. However, prior to progressing any such investigation, it was vital to seek guidance from those with existing expertise arising from their work with people who are Deafblind in the context of providing clinical and other support services.

This study therefore had two main objectives: 1) to develop an initial framework to engage Deafblind people in research and consultations on issues of public policy and service provision; and 2) to gather specific insights into how to conduct a World Café for Deafblind people. Consequently, the following research questions were addressed:

(1) What is current accepted practice in arranging events for, and consulting with, people who are Deafblind?

(2) How best can sign language and interpreter services be prepared for involvement in consultation and research activities, and more specifically as part of a World Café consultation?

(3) What knowledge, skills, tools and resources are needed when planning a World Café consultation for people who are Deafblind? 


\section{Method}

\section{Ethics}

This research was approved by the Human Research Ethics Committee of The University of Melbourne, with the application being supported by Able Australia (a specialist service provider for people with vision impairment).

\section{Design of the study}

Given the lack of existing information on the topic of inquiry, an exploratory design was adopted. The focus was on gaining insights and generating new ideas that would contribute to the formulation of an approach to consulting with people who are Deafblind, to be tested in a subsequent study. Consequently, a purposive sample of participants recognized for their existing experience and expertise was recruited through services specialising in the support of people who are Deafblind. The participants were subsequently engaged in semi-structured interviews, and the data subject to inductive thematic analysis using the constant comparative method (Bryant \& Charmaz, 2007; Charmaz, 2000).

\section{Participants}

The inclusion criteria required participants to be professional staff currently providing services to people who are Deafblind and experienced in organizing events for people who are Deafblind. Consequently, eight professionals participated in this study, all of whom resided in the same metropolitan capital city, and all eight professionals had experience providing services and consulting with Deafblind people around Australia and internationally. The participants included two sign language interpreters, two orientation and mobility specialists, two Deafblind technology specialists, and two Deafblind event coordinators. Their years of experience ranged from 8 to 30 , with a mean of 19.5 years.

\section{Procedure}

Eight professionals in Australia were interviewed to gain insights into developing a good practice approach to consultations and research activities involving people who are Deafblind. Given that the Deafblind community is so small, it is common for research to occur with people with whom you have a prior relationship. The researcher did have prior working relationships with all eight professionals and this was acknowledged in the plain language statement. The participants were encouraged to reflect on this and asked only to participate in the study if they felt comfortable. Furthermore, all participants had access to debriefing support via their National Professional Associations.

The professionals, all of whom had prior knowledge and/or experience of focus groups, were told about how WCs are conducted and the theoretical underpinnings of appreciative inquiry. Examples of the information provided to the participants are given in Table 1. 
Table 1: How the elements of the traditional problem-solving model compare and contrast to those of Appreciative Inquiry.

\begin{tabular}{ll}
\hline Problem Solving Model & Appreciative Inquiry \\
\hline Problem defined & $\begin{array}{l}\text { Appreciating what we have is the best at this } \\
\text { point in time, but acknowledging it might not } \\
\text { meet our current needs }\end{array}$ \\
\hline Cause (s) specified & $\begin{array}{l}\text { Creating a vision of what might be the various } \\
\text { alternatives }\end{array}$ \\
\hline $\begin{array}{l}\text { Solutions generated to address causes, and } \\
\text { by implications the problem }\end{array}$ & $\begin{array}{l}\text { Engaging in a dialogue to agree on what } \\
\text { should be, given the various alternatives }\end{array}$ \\
\hline $\begin{array}{l}\text { Actions specified to implement the identified } \\
\text { solutions }\end{array}$ & \begin{tabular}{l} 
Innovating to achieve the shared goal \\
\hline
\end{tabular} \\
\hline
\end{tabular}

The WC has many of the characteristics of a focus group, in that a group of people come together and are asked rounds of questions and are encouraged to interact when discussing their responses to the questions. However, the WC format has some unique characteristics. The overall environment should attempt to resemble a café style atmosphere (e.g. food, coffee and tea, waiters, decorative table settings), which is asserted to enhance the quality of the discussion. Furthermore, the WC typically consists of three rounds of research questions, between which people move from one group to another to promote the sharing and cross pollination of ideas. However, one participant from each group remains at a table to 'host' the next group and share the insights that were generated from the previous round.

The participants were told about how appreciative inquiry informs the following seven identified principles and processes of WC methodology (Brown, 2018): (1) Set the context- define the purpose, target the right people, establish the 'beginning questions'; (2) Create Hospitable Space - attend to the needs and comfort of participants; (3) Explore Questions that Matter explore real life concerns of relevance to the participants; (4) Encourage Everyone's Contribution - people are not simply participants, but contributors, so develop rules and moderate group behavior to maximize everyone's participation; (5) Connect Diverse Perspectives - get people moving and exchanging ideas with others with whom they might not ordinarily converse; (6) Listen together for Patterns and Insights - encourage people to listen to each other, and for what is not being spoken (forgotten or held back by social convention) along with what is being shared; (7) Share Collective Discoveries - throughout the process of the small group conversations and at the 'harvest', where the various conversations are distilled by the group as a whole, to generate proposed new directions.

In the current context, the participants were asked to consider how researchers could maintain fidelity to the established practices and processes of WC but remain open to the adaptation of the approach when considering the communication, technology, orientation and mobility, psychological, social, and cultural needs of Deafblind participants.

An interview schedule was designed with six open-ended questions, which enabled the participants to provide complete descriptions about their work with Deafblind people (see Table 2). Some general prompts to expand on answers, for example participants were asked; "can you tell me more?". All interviews were audio recorded and lasted between 30-45 minutes. The audio recordings were transcribed into NVivo version 11 by the first author. 


\section{Table 2: Interview Questions}

\section{A) Event Coordinator Individual Interviews}

1. Please describe the Deafblind events that you organize.

2. How long have you been organizing events like this (years)?

3. What is your professional background, training and qualifications?

4. Based on your experience, what knowledge and skills do you need to plan and implement a Deafblind event; and anything in particular with reference to a World Café?

5. What resources and tools are needed to facilitate a Deafblind event; and anything in particular with reference to a World Café?

6. If you were to advise researchers who are about to plan a Deafblind event, designed to consult with and elicit the views and opinions of people who are Deafblind, what would you tell them?

\section{B) Technology Specialist Individual Interviews}

1. Please describe the Technology Services you provide to people who are Deafblind.

2. How long have you been providing services like this (years)?

3. What is your professional background, training and qualifications?

4. Based on your experience, what knowledge and skills do you need to plan for and address the Technology needs of people who are Deafblind; and anything in particular with reference to a World Café?

5. What resources and tools are needed to address the Technology needs of people who are Deafblind; and anything in particular with reference to a World Café?

6. If you were to advise researchers who are about to plan a Deafblind event, designed to consult with and elicit the views and opinions of people who are Deafblind, what would you tell them?

\section{C) Orientation and Mobility Specialist Individual Interviews}

1. Please describe the Orientation \& Mobility Services you provide to people who are Deafblind.

2. How long have you been providing services like this (years)?

3. What is your professional background, training and qualifications?

4. Based on your experience, what knowledge and skills do you need to plan for and address the Orientation \& Mobility needs of people who are Deafblind; and anything in particular with reference to a World Café?

5. What resources and tools are needed to address the Orientation \& Mobility needs of people who are Deafblind; and anything in particular with reference to a World Café?

6. If you were to advise researchers who are about to plan a Deafblind event, designed to consult with and elicit the views and opinions of people who are Deafblind, what would you tell them?

\section{D) Interpreting Consultant Individual Interviews}

1. Please describe the Interpreting Services you provide to people who are Deafblind.

2. How long have you been providing services like this (years)?

3. What is your professional background, training and qualifications?

4. Based on your experience, what knowledge and skills do you need to plan for and address the Interpreting needs of people who are Deafblind; and anything in particular with reference to a World Café?

5. What resources and tools are needed to address the Interpreting needs of people who are Deafblind; and anything in particular with reference to a World Café?

6. If you were to advise researchers who are about to plan a Deafblind event, designed to consult with and elicit the views and opinions of people who are Deafblind, what would you tell them? 


\section{Interview analysis}

Data were analysed by the first author using inductive thematic analysis, applying the constant comparative method (Fram, 2013; Grove, 2010). Themes were recorded as 'free nodes'. Coded material was organized into overarching themes. Text queries were conducted on key words such as "Deafblind culture" and "communication" to ensure that all the relevant data were allocated to the nodes. Memos were used to help expand the process of inductive thematic analysis (Birks, Chapman \& Franic, 2008). These results were subsequently reviewed by the co-authors, with any discrepancies in coding resolved by consensus.

\section{Results}

The analyses revealed six major themes: (1) acknowledging the unique Deafblind world view; (2) the interplay of vulnerability and trusting relationships; (3) the importance of specialist knowledge, skills, and cultural sensitivities; (4) power to the people - addressing power imbalances inherent in consultation processes; (5) same but different - the Deafblind experience of being a CALD community; and (6) a challenge that must be met - the imperative to involve people who are Deafblind in research and policy development. These themes are presented below. Their subsequent implications for the development and implementation of an initial good practice framework to engage Deafblind people in research and policy development are then addressed in the discussion with specific reference to the three research questions.

\section{Theme 1 - Acknowledging the unique Deafblind world view}

Drawing on their professional experience, participants reported that Deafblind people share similar opinions regarding a lack of exposure to broader concepts and incidental learning due to multiple accessibility barriers to a sighted and hearing world. Six professionals specifically noted that Deafblind people are 'not always exposed to the same type of information than the wider community'. One professional stated, 'Deafblind people are often navigating information in the here and now and are more adept at trying to understand what immediately affects them'. Another stated, 'Deafblind people can experience difficulties understanding the big picture, so their world view, knowledge and experience is different'.

Although there was consensus between the professionals that Deafblind people have a reduced world view, this reduced world view appeared to be directly linked to the Deafblind person's inability to access information as opposed to cognitive impairments or an intellectual inability to grasp boarder concepts. For those people who acquire Deafblindness later in life, the professionals noted that people with acquired Deafblindness are still impacted by communication deprivation that in effect impacts their ability to access the world, ultimately impacting their world view. All eight professionals suggested that communication and meaning making needs to be framed in a unique way 'so as to help the Deafblind person have access to contextual meaning and a holistic view'.

\section{Theme 2 - The interplay of vulnerability and trusting relationships}

Another theme that emerged from the data was the mutual and dynamic nature of vulnerability and trust that occurs between Deafblind people, professionals and their physical environment. Four professionals identified concepts of vulnerability and trust consistently throughout their interviews. One professional noted, 'It's about developing that trust between the instructor and Deafblind client, it's important to make people feel safe in the environment'. Another professional noted, 'you're in physical contact with people a lot in this job and it's really important that you're respectful of their space'. Reflective questions were encouraged to help bring vulnerability and trust building issues to the surface. 
One professional noted witnessing people 'who aren't comfortable, or they feel a bit nervous if they're having to guide someone, they'll grab their hand and kind of just drag them through the environment or grab their hand and put it on something which is really invasive for those people'. She built on this notion of vulnerability and trust articulating the importance of 'hand under hand' which is a very respectful way of guiding 'that also gives that person choice to not participate in that activity which is really important when building up that trust'. And same with the guiding, there's ways of guiding people, that again, it allows that person being guided to let go of your arm or to refuse or not have that engagement if they don't want too'. This professional acknowledged 'it can be a bit of a scary field if people have no language skills to communicate in that space'. One professional identified the sense of when vulnerability and trust come together, 'there appears to be a connection, Deafblind people and professionals can relate, instead of operating out a sense of confusion or inadequacy, there's this lovely mutuality that's there'.

\section{Theme 3 - The importance of specialist knowledge, skills, and cultural sensitivities}

Building on the theme of "vulnerability and trust" a consistent observation that emerged across all professional interviews was "specialist skills to connect" with the Deafblind community. One professional noted 'there is a varied nature of Deafblind experience and a myriad of needs; interpreters act as professional conduits who need experience of Deafblind culture and language to smoothing out cultural difference'. Furthermore, all professionals noted the 'unique linguistic world conveying meaning and communication from verbal, to visual and even tactile language'. They all highlighted the importance of providing contextual links, examples and taking time to unpack meaning with Deafblind people. Professionals identified that Deafblind communication requires touch, connection, patience, flexibility and the ability to respond to diversity, awareness of cultural nuances, and respect for client idiosyncrasies. All eight professionals referred to the spectrum of Deafblind people and the importance of trying to understand and connect with their lived experience.

\section{Theme 4 - 'Power to the people' - addressing power imbalances inherent in consultation processes}

Another theme that emerged was the importance of working in ways that included antioppressive and empowerment perspectives. All professionals displayed an acute awareness of power dynamics between hearing professionals and Deafblind people. All participants shared examples of situations where Deafblind oppression and marginalization could occur and specific insights into how they navigate power imbalances within their own practice. For example, one professional identified as 'being a dominant person' and pointed out that research has found that people without disabilities dominate conversations by speaking more freely than their disabled peers. This professional spoke of being aware of her position within the conversation and consciously creating a mutuality with the Deafblind person. Another professional stated that he plans to design a conference for Deafblind people that is experiential and tactile so that Deafblind people can experience as much as they can independently; this type of experience 'allows them to have more control about what's happening without relying on an interpreter; allowing them to experience first-hand'. Several professionals analysed Deafblind oppression and marginalization within a historical framework. For example, one professional identified the power issues that exist when multiple people are speaking at the same time. 'For an interpreter to stop someone for signing, and tell them hold, someone else is talking; for someone who hasn't worked with many Deafblind people they are reluctant to hold and tell them to stop signing. This goes back to history of control and man handling, telling them when they can and can't speak. It's hard for an interpreter to do but very much needed in Deafblindness'. 
Professionals identified the unique ethical challenges that exist within the Deafblind community in relation to 'the fine line that exists between trying to provide context and meaning versus offering up leading questions and examples which may influence the Deafblind person'. One interpreter highlighted that there is a tension between unpacking questions to help the Deafblind person understand and actually dangerously leading and influencing the Deafblind person's answers; 'For an interpreter to unpack and add meaning and to be sure it is understood we give examples, such as are you happy most of the time or sad sometimes? But these questions are all leading the client. The Deafblind person may never have thought like that and I ended up leading them. I know that in a lot of areas of interpreting unpacking questions is a dangerous. We often ask the speaker to give an example, I don't want to lead the client or give them ideas.

Another professional identified Deafblind oppression and marginalisation from access to participation and inclusion. She stated that 'the Deafblind community typically view an event as somewhere to gather information' as opposed to contributing and sharing their lived experiences. Moreover, she claimed that Deafblind people 'are commonly not used to being asked their opinion or feeling safe to share their needs and ideas'. Interestingly, she identified that the Deafblind community 'are still learning how to form a viewpoint and give an opinion in a group setting. It takes a lot of trust in the group to venture opinions and I believe this is still developing'. Overall, professionals appeared to have an awareness of Deafblind oppression and marginalisation with an historical context and a unique awareness of their own sense of power within Deafblind interactions.

\section{Theme 5 - Same but different - the Deafblind experience of being a CALD community}

The theme 'Deafblind subculture' was induced from the responses of all eight professionals working in the Deafblind sector. The professionals placed Deafblind people within the dominant Australian context suggesting that they have exposure to mainstream beliefs, values, history and norms. However, the professionals noted that Deafblind people (and in particular Auslan and tactile users) may experience (and form) a unique subculture within the larger society, although one with its own distinct heterogeneity. The Deafblind subculture, though identified as being a cohesive entity, was characterised as being a unique culturally and linguistically diverse community regarding experiences of touch, physical space and connection. For example, one hearing professional noted, 'there is a core group of Auslan and tactile users, there is a very strong cultural self-conception around the Deafblind community'. A Deaf professional highlighted, 'It is very important to understand, well as you know, Deaf and hearing people have their own culture. Many Deafblind people consider themselves belonging to another culture again'. This same professional identified the importance of touch and connection within the Deafblind community. 'Touch is a key for Deafblind people. I would have a person sitting beside me who I could physically have contact with, with my elbow or my arm to know that that person is there so it builds a relationship, a connection'.

Six professionals identified a unique Deafblind subculture comprised of a diverse spectrum of people who experience Deafblindness, and a community of professionals such as interpreters, communication guides, orientation and mobility specialists and allied health. One hearing professional who identified as being more of an 'outsider' to the Deafblind community remarked, 'these people are not just professionals but cultural advocates, bridges and allies'. She went on to comment on 'richness of perspective and experience and that the Deafblind community isn't just people with Deafblindness, you know that there's this whole community that share these amazing communication skills, it's a shared thing, they connect, and they know how to relate to Deafblind people'. Four professionals identified 'cultural immersion' as an important factor for outsiders to the Deafblind community. For example, another professional noted 'with any minority linguistic group, it is very important for a hearing person or a researcher who doesn't know this linguistic world, like sign language for example, it's 
important to have some kind of immersion an experiential workshop so you have some feeling or some experience of what it would be like to be Deafblind'.

A minor theme related to Deafblind subculture that emerged was the experience of marginalisation within the Deafblind community; between those who use Auslan and tactile sign compared to those people with Deafblindness who use speech. Three professionals noted that the Deafblind subculture is comprised predominately of Deafblind people who identify as being part of a culturally and linguistically diverse minority group as opposed to a disability. One participant noted; 'there is a core group of people who use Auslan who attend most things, a lot of people (who only use speech) might pull back from being involved, because historically they feel like it's not their place'. I guess what I would call these people outlining individuals in this community. It tends to be the people who I would say don't identify culturally as strongly with the Deafblind community'. Several professionals appeared to be aware of the marginalisation within the Deafblind community of those who only use speech, one professional highlighted that those who only use speech form an 'important and very valid perspective on the Deafblind experience which often gets left out of'.

\section{Theme 6 - A challenge that must be met; the imperative to involve people who are Deafblind in research and policy development.}

All eight professionals argued that Deafblind people have a diverse range of skills, expertise and experiences that can contribute to research, policy, support and service development. Even so, 'historically Deafblind people have been silenced' (Deafblind interpreter) and have 'few opportunities to participate in formalised research' (Deafblind manager). There was strong consensus that Deafblind people want to be included in research and consultation processes, and in order to do so, specific adaptations regarding technologies, resources, orientation and mobility, communication, linguistic considerations need to be made. Furthermore, the professionals in the field of Deafblindness provided more specific, comprehensive and practical ideas on how to adapt the traditional World Café methodology and make it appropriate for Deafblind people. Consensus was evident among all participants in this study that Deafblind people may be even more sensitive to 'creating a hospitable space' and that psychological and physical safety needed to be considered (regarding managing emotions, personalities, communication styles, ordination and mobility and support needs).

Regarding Deafblind World Café etiquette, the majority of participants identified that the host/researcher would need to converse with Deafblind people and their communication supports throughout the methodological process thus acting as a cultural bridge between the world of research and the Deafblind community. All the professionals made specific reference to providing context, examples and information to help Deafblind people understand the purpose of the World Café methodology. The participants argued that researchers cannot assume that Deafblind people have had any exposure to research terminology and processes or consultation activities (regardless of their cognitive abilities). Researchers need to consider Deafblind people's lack of incidental learning, sensory and communication deprivation, and design research programs that can respond to the heterogeneity of Deafblind people, provide opportunities for education, and empower and capture their lived experiences.

\section{Discussion}

This study was conducted as part of a program of research designed to establish a trustworthy method and supporting technologies to effectively engage with those who are Deafblind in order to inform research, policy and practice. Critically, it was considered important that any such process should be respectful of people who are Deafblind, recognising the diversity of such individuals but that they form a distinct cultural community bound by language and customs of their own. Moreover, given the absence of guidelines on how this might be 
achieved, a consultation process involving professionals who currently work with people who are Deafblind in clinical and support settings was considered important.

Analyses of the semi-structured interviews conducted with purposively sampled experts generated six themes, as outlined above. These themes and their sub-categories were used to answer the three research questions posed for the current study.

(1) What is current accepted practice in arranging events for, and consulting with, people who are Deafblind?

There was consensus that there are no current formalized best practice guidelines regarding arranging events for and consulting with people who are Deafblind. The professionals placed importance on experiential learning, and cultural and linguistic immersion with the Deafblind community, as opposed to relying on formalized guidelines, courses or training programs. These data revealed that to arrange events and consult with Deafblind people, professionals must have an awareness of the Deafblind subculture and a deep understanding of the heterogeneous nature of Deafblind communication. The notion of professional boundaries and how to work with them also emerged as an important consideration. Notably, the professional relationships that were discussed did not represent the traditional 'client-service provider dynamic'. For example, the Deafblind and professional relationship was characterised by the involvement of physical touch and sharing of a unique language and culture. Relationships (such as the role of a support worker and communication guide) emerged as complex; they were underpinned by funding, could span decades, and survive many socio-political and economic challenges. An awareness of one's own power, and the potential for Deafblind oppression and marginalisation, was central to the thinking of all the professionals interviewed.

(2) How best can sign language and interpreter services be prepared for involvement in consultation and research activities; and more specifically as part of a World Café consultation?

The data revealed few specific recommendations regarding how sign language and interpreter services could be best prepared for Deafblind consultation and research activities. However, the interpreters provided specific recommendations to help researchers be better equipped to research and consult with Deafblind people. These included issues associated with the construction and deconstruction of language, the dynamic role of the Deafblind interpreter, ethical considerations, and cultural and linguistic sensitivity.

Regarding the construction and deconstruction of language, one interpreter suggested 'Take your time; unpack the language so we don't have to'. They urged researchers to 'consult with interpreter; do you have any ideas how I can get this question thru?' This participant was indicating that the interpreter profession has gone through a transition from a 'conduit method, which only relayed communication back and forward' to current accepted practice which allows Deafblind interpreters to be a be a linguistic and cultural bridge. For example, 'we have lots of experience smoothing out a cultural difference and bridging a gap between language or culture, understanding or concept'. The interpreters requested to have license from the researchers to unpack the questions, provide examples and scenarios to help with Deafblind comprehension 'If my intention is right then I am not breaking any ethics by consulting with you the professional, I have an idea, can you rephrase the question this way, does it have the same meaning?' The interpreters in this study recommended that research teams needed to value diversity and have an awareness of accessibility issues based on an experiential awareness and appreciation of the unique cultural and linguistic world of the Deafblind community. 


\section{(3) What knowledge, skills, tools and resources are needed when planning a World Café consultation for people who are Deafblind?}

In order to conduct large scale face-to-face consultation processes (such as the World Café) with a heterogeneous group of Deafblind people, a range of theoretical and practical skills, tools and resources need to be employed. Prior to embarking on research, a team of specialists should be engaged for their expert knowledge in interpreting, translation and communication, disability support coordination, orientation and mobility, assistive technology and advocacy. Methodological experts (such as a World Café expert) should be consulted to help address the inherent challenges in adapting mainstream and traditional research methodologies for people with limited vision and hearing. Here, it is critical that there is culturally sensitive adaptation of traditional methods, while the validity and reliability (trustworthiness) of such methods is maintained.

When planning an event (such as the World Café) researchers and practitioners alike must understand that the Deafblind person may experience a reduced world view due to sensory deprivation and as a result of limited exposure to concepts of research. However, researchers must not assume that communication deprivation equates to psychopathology, intellectual disability or the inability to participate in consultation, research and policy development (Mathos et al., 2011; Vernon, 1982, 2010). Deafblind people should be central in the coproduction and co-design of the research project. However, the research suggests that the experience of Deafblindness is one of exclusion, and that the nature of being Deafblind is so isolating that for practitioners, clinicians and policy makers alike it can be considered a group whom it is too difficult to consult. Subsequently, lack of consultation means that contemporary ideas such as co-design and co-production and working collaboratively in partnership can be easily disregarded and result in paternalistic practice in which the so-called professionals and "gatekeepers of knowledge" know best. Many of these 'wicked problems' still need addressing for Deafblind people to have an empowered voice within the socio-political arena. In order to do so, the researcher needs to spend time with the community, developing relationships and preparing Deafblind people for the research experience (e.g. in the form of workshops, community briefings, accessible tools, and information prior to the event). Researchers would benefit from cultural immersion by gaining insider knowledge of the Deafblind person's culture and community.

Research should ideally be conducted in spaces known to the Deafblind community due primarily to the practicalities and safety of orientation and mobility, but also to promote cultural ownership of the project. By developing mutual relationships of vulnerability and trust (built on touch, communication and time), the researcher and Deafblind person can develop skills to explore questions that matter, and share lived experiences. Deafblind people want to be included in consultation, research and policy processes but to do so, adaptations need to be made (Heine \& Browning, 2012). The 'wicked problem' can be addressed when Deafblind people are supported to co-author the research agenda and develop subsequent solutions to their own lives.

\section{Issues around methodology}

The findings of the current study are based on interviews with a relatively small group of participants. However, given they were purposively sampled for their professional experience and expertise, and the extent to which they were in agreement with respect to the various issues (in many instances all eight contributed data to each of the emerging themes) supports the assertion that both data saturation and theoretical saturation were achieved within the limited data set. All eight participants worked predominantly in a single Australian state and all had experience of working with the Deafblind community across Australia (e.g., being involved in national conferences). Despite these limitations, this study usefully provided background 
information regarding how to consult with and elicit experiences from people who are Deafblind in ways that are culturally appropriate and technically reliable.

\section{Conclusions}

In seeking to investigate good practice approaches to consultation and research activities for people with Deafblindness, much can be gained from examining the findings of this study, particularly in regard to how to enhance the inclusion of people from CALD and minority groups in consultation, research and policy development. Furthermore, this study challenges the inherent assumptions of living in a hearing and sighted world that are still evident within current inclusive research paradigms. Deafblind people, like those of other CALD and minority groups (e.g. Deaf and Blind), experience complex communication and sensory deprivation that impacts their access to information and their ability to participate in the society that ultimately shapes their world view (Selepak, 2008). As a result of the pervasive nature of Deafblindness, people with limited sight and sound navigate complex dynamics of vulnerability and trust within both their environment and relationships on a daily basis (Asherman, 2012; Barrett, 1992; Berry et al., 2008). A host of specialist skills are required to connect with Deafblind people so that they can express their full potential and share their lived experience (Blumsack, 2009). Anti-oppressive and empowerment perspectives and cultural immersion are essential for the scientific community to grasp in order to consult with and plan events for Deafblind people and those who identity with CALD and minority groups.

The field of Deafblind research is largely underdeveloped; Deafblind people want to be included in research and consultation processes but to do so, researchers and professionals alike need to deploy accessible and culturally sensitive research methodologies that are reliable and valid (Asherman, 2012). Subsequent studies need to place Deafblind people as experts of their lived experience and the co-producers of research methodologies, consultation processes and research activities. Moreover, a specialised research tool kit and research guidelines designed by Deafblind people are needed to ensure good practice approaches to research and consultation activities with Deafblind people. Finally, qualitative methodologies such as World Café may be effective in providing a safe environment for people with diverse cultures, identities and abilities to share their experience and co-produce the theoretical, technical, and practical components needed for inclusive research and policy development processes (MacFarlane et al., 2017; Merier \& Geldenhuys, 2017; Selepak, 2008).

\section{Author note}

This study was completed in partial fulfilment of a Doctor of Philosophy candidature by the first author under the supervision of the second and third authors. No restrictions were placed on free access to or publication of, the research data. No financial benefit to the authors arises from the publication of this paper.

\section{Acknowledgements}

We knowledge the diverse range of skills and experiences of the experts in the field of Deafblindness and express gratitude for their participation in this study. 


\section{References}

Alford, J. (2014). 'The Multiple Facets of Co-Production: Building on the work of Elinor Ostrom'. Public Management Review, 16 (3), 299-316.

Asherman, J, J. (2012). Culturally affirmative mental health recovery program for deaf, hard of hearing, deaf-blind and late-deafened individuals: A grant proposal California State University, Long Beach, 64; 1511330.

Australian Institute of Health and Welfare (AlHW) (2011). Aboriginal and Torres Strait Islander people with disability. Cat. no. IHW 45. Canberra: AIHW.

Barrett, S. (1992). 'Comprehensive Community-Based Services for Adults Who Are DeafBlind: Issues, Trends, and Services'. Journal of Visual Impairment and Blindness, 86 (9), 393-397.

Beyea, C.S. \& Nicoll, H. L. (2000). 'Collecting, analyzing, and interpreting focus group data'. AORN Journal. 71.6 (June 2000), 1278.

Berry, P., Kelley-Bock, M. \& Reid, C. (2008). 'Confident living program for senior adults experiencing vision and hearing loss'. Care Management Journals, 9 (1), 31-35.

Billson, J. M. (2004). The Power of Focus Groups: A Training Manual for Social and Policy Research: Focus on International Development (4th edition), Barrington, RI: Skywood Press.

Birks. M., Chapman. Y. \& Franic. K. (2008). 'Memoing in qualitative research; probing data and process. Journal of Research in Nursing'. VOL 13(1) 68-75.

Bloeming-Wolbrink, K. A., Janssen, M. J., de Weerth, C., Ruijssenaars, W. A. J. J. M., Sweep, F. C. G. J., Eijsbouts, A. \& Riksen-Walraven, J. M. A. (2012). 'Stress in adults with congenital deafblindness and an intellectual disability: Information from their cortisol curves'. British Journal of Visual Impairment, 30 (3), 149-159.

Blumsack, J. T. (2009). 'Dual Sensory Loss: A Guide for Outreach to Primary Care Physicians'. Seminars in Hearing, 30 (3), 207-221.

Bodsworth, S. M., Clare, I. C. H. \& Simblett, S. K. (2011). 'Deafblindness and mental health: Psychological distress and unmet need among adults with dual sensory impairment'. British Journal of Visual Impairment, 29 (1), 6-26.

Bonevski, B., Randell, M., Paul, C., Chapman, K., Twyman, L., Bryant, J., Brozek, I. \& Hughes, C. (2014). 'Reaching the hard-to-reach: a systematic review of strategies for improving health and medical research with socially disadvantaged groups'. BMC Medical Research Methodology, 1.

Bovaird, T. \& Loeffler, E. (2010). 'User and community co-production of public services and public policies through collective decision-making: the role of emerging technologies', in T. Brandsen and M. Holzer (eds), The Future of Governance. Newark, NJ: National Center for Public Performance.

Brennan, M., Su, Y.-p. \& Horowitz, A. (2006). 'Longitudinal associations between dual sensory impairment and everyday competence among older adults'. Journal of Rehabilitation Research And Development, 43(6), 777-792.

Brown, J. \& Isaacs, D. (2005). 'The World Café Community'. In The World Cafe (pp. 96-98). Berrett-Koehler Publishers.

Brown, J., Issacs, D. \& Tan, S. (2008). 'Multi-generational collaboration: Shaping tomorrow, together'. Kosmos, 7 (2), 32-33.

Brown, S. J. (2018). World Café. Retrieved 08/08/18 from http://www.theworldcafe.com/aboutus/.

Bruce, M. S., \& Parker, T. A. (2012). 'Young Deafblind Adults in Action: Becoming SelfDetermined Change Agents Through Advocacy'. American Annals of the Deaf, 157(1), 16-26.

Bryant, A. \& Charmaz, K. (2007). The SAGE handbook of grounded theory: SAGE Publications Ltd. 
Buckmaster, L. \& Thomas, M. (2009) 'Social inclusion and social citizenship towards a truly inclusive society', Research Paper No. 08 2009-10. Social Policy Section, Commonwealth of Australia.

Bumble, L. J., Carter, W. E., McMillan, E., Manikas, S. A., \& Bethune, K. L. (2018). 'Community Conversations on Integrated Employment: Examining Individualization, Influential Factors, and Impact'. Journal of Disability Policy Studies, 28(4), 229-243.

Burke, C. \& Sheldon, K. (2010). 'Encouraging workplace innovation using "World Cafe" model'. Nursing Management, 17 (7), 14-19.

Carson, L. (2011). 'Designing a public conversation using the world café method'. Social Alternatives, 30(1), 10-14.

Carter, E. W., Swedeen, B., Cooney, M., Walter, M. J. \& Moss, C. K. (2012). “I don't have to do this by myself?": Parent-led community conversations to promote inclusion'. Research and Practice for Persons with Severe Disabilities, 37, 9-23.

Carter, E. W., Austin, D., \& Trainor, A. A. (2012). 'Predictors of postschool employment outcomes for young adults with severe disabilities'. Journal of Disability Policy Studies, 23, 50-63.

Carter, E., Sweeden, B. \& Molfenter, N. (2010). 'Getting everyone involved: Identifying transition opportunities for youth with severe disabilities'. Teaching Exceptional Children, 43, 38-49.

Charmaz, K. (2000). 'Grounded theory: objectivist and constructivist methods'. In: N. K. Denzin \& Y. S. Lincoln (eds.), Handbook of Qualitative Research (pp. 509-536). Sage: London.

Churchman, C. W. (1967). 'Wicked Problems (Guest Editorial)'. Management Science. 14 (4).

Clark, M. J., Cary, S., Diemert, G., Ceballos, R., Sifuentes, M., Atteberry, I., Vue, F. \& Trieu, S. (2003). 'Involving communities in community assessment'. Public Health Nursing 20, 456-463.

Dutta, M. J. (2007). 'Communicating about culture and health: Theorizing culture-centered and cultural sensitivity approaches'. Communication Theory, 17, 304-328.

European Parliament. (2004). Declaration of rights of deafblind people. Retrieved August 29, 2018 http://www.europarl.europa.eu/sides/getDoc.do?pubRef=-//EP//TEXT+TA+P5$\mathrm{TA}-2004-0277+0+\mathrm{DOC}+\mathrm{XML}+\mathrm{V} 0 / / \mathrm{EN}$.

Fallon, H. \& Bean, E. (2014). 'Hosting a world cafe: Experiences at the National University of Ireland Maynooth'. SCONUL Focus, 60, 72-74.

Ferlie, E., McGivern, G., Fitzgerald, L., Dopson, S. \& Bennett, C. (2011) 'Public Policy Networks and 'Wicked Problems': A Nascent Solution?'. Public Administration, 89 (2) 307-324.

Fram, S. M. (2013). 'The Constant Comparative Analysis Method Outside of Grounded Theory'. The Qualitative Report, 18(1), 1-25.

Grove, W.R. (2010). 'An analysis of the constant comparative method'. International Journal of Qualitative Studies in Education Volume 1, 1988 - Issue 3.

Gulanick, M. \& Keough, V. (1997). 'Focus Groups; an exciting approach to clinical nursing research'. Prog Cardiovasc Nursing, 12(2), 24-29.

Hammel, J., Magisi, S., Heinemann, A., Whiteneck, G., Bogner, J. \& Rodriguez, E. (2008). 'What does participation mean? An Insider perspective from people with disabilities'. Disability and Rehabilitation, 30(19), 445-1460.

Heine, C. \& Browning, C. J. (2002). 'Communication and psychosocial consequences of sensory loss in older adults: overview and rehabilitation directions'. Disability and Rehabilitation, 24(15), 763-773.

Heine, C. \& Browning, C. J. (2014). 'Mental health and dual sensory loss in older adults: a systematic review'. Frontiers in Aging Neuroscience, 6, 1-9.

Hillier, L., Turner, A. \& Mitchell, A. (2005). Writing Themselves in Again: 6 Years On: The 2nd National Report on the Sexual Health and Well-Being of Same Sex Attracted Young People in Australia. Monograph Series. No. 50 (Australian Research Centre for Sex, Health and Society, La Trobe University Melbourne). 
Horvath, B. (2005). 'Building on traditions of dialogue: Saudi Aramco'. In J. Brown and D. Isaacs (eds.), The world cafe: Shaping our futures through conversations that matter (pp. 98-109). Berrett-Koehler, San Francisco, CA.

Hung, L., Phinney, A., Chaudhury,H., Rodney, P., Tabamo, J. and Bohl,. D. (2018). 'Appreciative Inquiry: Bridging Research and Practice in a Hospital Setting'. International Journal of Qualitative Methods, 17 (1), 1-10.

Jasanoff, S. (2004). States of Knowledge: The Co-Production of Science and Social Order. Routledge, London, UK.

MacDonald, R. J. (1985). 'On gaining independence as a deaf-blind person'. Journal of Visual Impairment \& Blindness, 79(5), 205-208.

MacFarlane, A., Galvin, R., O'Sullivan, M., Mclnerney, C., Meagher, E., Burke, D. \& LeMaster, J.W. (2017). 'Participatory methods for research prioritization in primary care: an analysis of the World Café approach in Ireland and the USA'. Family Practice, 34, (3) 278-284.

Mathos, K., Lokar, F. \& Post, E. (2011). 'Gathering perceptions about current mental health care services and collecting ideas for improved service delivery for persons who are deaf, deafblind and hard of hearing'. Journal of the American Deafness and Rehabilitation Association, 44(3), 134 -152.

Meier, C. \& Geldenhuys, D.J. (2017). 'Co-constructing Appreciative Inquiry across disciplines: A duo-ethnography'. SA Journal of Industrial Psychology, 43, a1400.

Minkler, M. \& Wallerstein, N. (2012). 'Improving health through community organization and community building: Perspectives from health education and social work'. Community Organizing and Community Building for Health and Welfare, 37-58.

Muñoz-Erickson, T.A. (2014). 'Co-production of knowledge-action systems in urban sustainable governance: the KASA approach'. Environmental Science and Policy 37 (3), 182-191.

Needham, C. \& Carr, S. (2009). 'SCIE Research briefing 31: Co-production: an emerging evidence base for adult social care transformation'. Social Care Institute for Excellence, London, UK. https://www.scie.org.uk/publications/briefings/briefing31/.

Ostrom, V. \& Ostrom, E. (1978). 'Public goods and public choices', in E. Savas (ed.). Alternatives for Delivering Public Services. Toward Improved Performance. Boulder, CO: Westview Press, pp. 7-49.

Pawlikowska, T., Leach., J., Lavallee P., Charlton, R. \& Piercy, J. (2007). 'Consultation models'. In: J. Charlton (Ed), Learning to consult. 2nd ed. Oxford: Radcliffe Pub, pp.178 - 215.

Pranee, L. (2016). Focus group methodology: principles and practice. London. SAGE. 225.

Rittel, H., \& Webber, M. (1984). 'Dilemmas in a General Theory of Planning'. Policy Sciences, 4, 155-169. Elsevier Scientific Publishing Company, Inc., Amsterdam, 1973. Reprinted in N. Cross (ed.), Developments in Design Methodology, J. Wiley \& Sons, Chichester, 1984, pp. 135-144.

Saunders, P. (2013). 'Reflections on the Concept of Social Exclusion and the Australian Social Inclusion Agenda'. Social Policy and Administration, 47 (6), 692-708.

Schieffer, A., Isaacs, D. \& Gyllenpalm, B. (2004). ,World Caféé part one'. World Business Accademy, 18(8), 2-16.

Selepak, L. (2008), 'Challenges Facing People with Disabilities from Culturally and Linguistically Diverse backgrounds (CaLD) Monograph'. Disability Services Commission WA.

Shakespeare, T. (2006). Disability Rights and Wrongs, Routledge: London.

Sharp, E. (1980). 'Toward a New Understanding of Urban Services and Citizen Participation: The Coproduction Concept'. Midwest Review of Public Administration, 14(2), 105-118.

Simm, J. (1998). 'Collecting and analysing qualitative data: Issues raised by the focus group'. Journal of Advanced Nursing, 28, 345-352.

Trainor, A. A. (2018). 'Community Conversation as a Method of Gathering and Analyzing Qualitative Data'. Journal of Disability and Policy studies, 29(1). 
Vernon, M. (1982). 'Considerations in Diagnosing Usher's Syndrome: RP and Hearing Loss'. Journal of Visual Impairment and Blindness, 76 (7), 258-261.

Vernon, M. (2010). 'Deaf-Blindness and Autistic Spectrum Disorder'. Journal of the American Deafness \& Rehabilitation Association (JADARA), 44 (1), 201-213.

Wahlqvist, M., Möller, C., Möller, K. \& Danermark, B. (2013). 'Physical and psychological health in persons with deafblindness that is due to Usher Syndrome Type II'. Journal of Visual Impairment and Blindness, 107 (3), 207-220.

Wittich, W., Watanabe, D. H. \& Gagné, J.-P. (2012). 'Sensory and demographic characteristics of deafblindness rehabilitation clients in Montréal, Canada'. Ophthalmic \& Physiological Optics: The Journal of the British College of Optometrists, 32 (3), 242251.

Wyborn, C. (2015). 'Co-productive governance: a relational framework for adaptive governance'. Global Environmental Change, 30, 56-67.

\section{Biographical Notes}

Alana Roy is a practising Psychologist, Mental Health Social Worker and a PhD student in the School of Social and Political Sciences, University of Melbourne, Vic. Australia.

Keith McVilly is a registered Clinical Psychologist and the Foundation Professorial Fellow for Disability \& Inclusion, in the School of Social \& Political Sciences at the University of Melbourne.

Beth Crisp is Professor and Discipline Leader for Social Work in the School of Health and Social Development, Deakin University, Geelong, Vic. Australia. 